\title{
Televisión y aprovechamiento escolar en los niños de primaria de Monterrey, Nuevo León
}

\author{
Francisco Javier Martínez Garza \\ Universidad Autónoma de Nuevo León
}

\begin{abstract}
A LA televisión se le ha atribuido la dualidad del bien y del mal. Algunos destacan los beneficios que ofrece a la sociedad, otros, enfatizan su impacto negativo. Los puntos de vista encontrados y la polémica poco interesan a los telespectadores. La
\end{abstract} amplia penetración lograda, es el más claro indicador de su aceptación.

En Monterrey, capital de Nuevo León, la televisión ha logrado penetrar prácticamente en todos los hogares. Un estudio de audiencias de televisión (Martínez Garza,1991), reporta que $98 \%$ de los hogares cuentan al menos con un aparato televisor; de los cuales, alrededor del $70 \%$ tienen más de un aparato receptor.

En esta ciudad es posible recibir la señal de siete canales de televisión: canal 2 (Monterrey), 4 (Televisión Azteca), 6 (Televisa), 8 (Televisión Azteca), 10 (Televisa), 12 (Multimedios Estrella de Oro, Monterrey), 28 (Gobierno del Estado), además el sistema de cablevisión instalado en gran parte de la ciudad ofrece alrededor de 20 canales más.

Pese a que el medio se ha arraigado enormemente en esta ciudad, es fecha en que los estudiosos de la comunicación no comenzamos a estudiar ni tan siquiera descriptivamente la participación que aquí tiene el medio.

El presente artículo ofrece un panorama de la participación de la televisión en el aprovechamiento escolar de los niños de primaria en esta ciudad ubicada en el noreste de la República Mexicana. 
$\mathrm{El}$ interés por el tema se originó después de que un matutino de Monterrey efectuó un estudio donde se puso de manifiesto la existencia de un bajo nivel académico en los niños de primaria del área metropolitana. ${ }^{1}$

El estudio de dicho medio informativo considera que la mayor parte de la culpa recae en los profesores, argumento que no puede ser sostenido por el tipo de investigación realizada. Además resulta injusto si de antemano no son estudiadas otras variables que puedan estar involucradas en el problema.

Tomando en cuenta que los niños de esta región utilizan la televisión tres horas en promedio, es posible considerar que el medio puede ser una de las variables intervinientes en el problema.

La presente investigación se propone determinar si ese tiempo ha contribuido en el bajo nivel académico mostrado por los niños de primaria en Monterrey y su área metropolitana. Para responder al planteamiento anterior se ha formulado la siguiente pregunta: ¿Tienen los niños que dedican diariamente más tiempo a ver latelevisión, promedios de calificación más bajos que quienes la ven menor cantidad de tiempo?

Para efectos del estudio será considerado "Mayor cantidad de tiempo" el equivalente a ver televisión por lapsos mayores a las $\mathbf{3}$ horas y media al día; por "Menor cantidad de tiempo" se entenderán periodos de tiempo inferiores a una hora al día. La investigación comprende información recabada en el área metropolitana de la ciudad de Monterrey, Nuevo León, México y los resultados pueden variar en relación con la temporalidad y la territorialidad.

\section{Los niños se apropian del aparato receptor}

Es prácticamente imposible que los niños se alejen por completo de la televisión, para ellos eso es sinónimo de un "castigo terrible". Además no podemos negar que el medio constituye sobre todo una importante fuente de información, util para su propia socialización. Luego mientras que por una parte se ha hecho imprescindible, por la otra, se ha convertido en un problema tanto para los padres de familia como para los maestros en las escuelas.

1 La investigación apareció en la primera página de la sección cultural del periódico El Nonte de la ciudad de Monterrey. Comprendió un total de 4 días: del 7 al 10 de abril de 1991. 
El uso y el gusto por la televisión se han incrementando en forma notable. Hay quienes suponen que este medio de comunicación masiva acapara la mayor parte del tiempo libre de los individuos hoy en día.

Algunos estudios, señalan que el niño es el miembro de la familia que mayor tiempo dedica a ver televisión. A la vez, de acuerdo con los expertos, ellos - los niños - son también los más vulnerables al medio.

En el libro La televisión y el niño, los autores opinan: "pocos pueden negar que los niños son seres extraordinarios, activosy encantadores (...) Si de ellos dependiera la elección de cómo alimentarse, es posible que su dieta estaría compuesta principalmente de pastelitos y dulces. La distribución de su tiempo estaría repartida seguramente entre jugar y estar sentados frente al televisor viendo todo tipo de programas" (Fernández Collado et al, 1986).

Esta preferencia de los niños para con la televisión se presenta desde sus primeros años. Leticia Mas menciona que este fenómeno se presenta debido a que los mismos padres orillan a los niños a permanecer mayor cantidad de tiempo frente al televisor.

La misma autora señala en su artículo que: "muchas madres de familia están ansiosas por que desaparezca de sus hijos la teleadicción y así mejoren sus hábitos de estudio y su conducta" (...) Ellas - menciona Mas Mares - culpan la mayoría de las veces a los programas que sus hijos ven, sin percatarse de su irresponsabilidad al utilizar la televisión como una niñera para sus hijos" (Mas Mares, 1990:11).

Marie Winn menciona casos de niños que utilizan la televisión hasta 54 horas por semana. Es decir, dedican un tercio de sus horas de vigilia a estarla viendo (Winn, 1981). José Carlos Lozano, menciona el caso de estudiantes de escuelas secundarias de Nuevo Laredo Tamaulipas - $200 \mathrm{kms}$ al norte de Monterrey - que utilizan la televisión en promedio de 242 minutos, es decir 4 horas diariamente (Lozano, 1992)

Otrode los factores que contribuyen para que el niñodedique grandes cantidades de tiempo a ver televisión es el ambiente mismo en el cual se desarrolla. Acosta en su artículo "Los niños mexicanos y la televisión", presupone la existencia de una relación directa entre el medio en el cual se desenvuelve el niño y el uso que hacen de la televisión (Acosta, 1972).

También Fernández Collado et al (1984) suponen que las costumbres del grupo social al cual pertenece la familia del niño influyen en su conducta y en sus necesidades desde temprana edad, de igual manera los hábitos del niño se verán influidos por las personas adultas que vivan con él, especialmente los padres. 
Orozco Gómez (1991:6) sostiene que el niño puede convertirse en un televidente creativo y crítico, capaz de discernir entre diversos tipos de mensajes, tomar distancia entre ellos y criticar y rechazar aquellos que así juzgue conveniente. Pero para que ello suceda dice, depende mucho del papel que jueguen la familia y la escuela frente al televisor, con respecto a la educación del niño televidente.

\section{El niño pasivo}

Los largos períodos de tiempo que dedica el niño a ver la televisión tal vez no serían de consecuencias negativas si el medio no originara ciertas conductas y hábitos en los infantes. Pero a decir de muchos especialistas, la televisión crea pasividad en el niño, misma que le impide realizar actividades propias de los pequeños como jugar, socializarse e incluso tener poco interés por sus clases y actividades escolares.

Entre los autores que afirman lo anterior se encuentra Winn (1981), quien sostiene que los niños necesitan adquirir ciertas habilidades fundamentales de comunicación: leer, escribir, y a expresarse flexiblemente y con claridad para poder funcionar como criatura social, lo cual a juicio de la autora, la televisión no mejora su desarrollo verbal, porque no le exige ninguna participación verbal, sino una mera absorción pasiva.

Al igual que Winn existen otros estudiosos del tema que sostienen que la misma televisión induce al niño a una especie de letargo, donde se presenta la apatía para realizar cualquier otro tipo de actividad. Vallejo-Nagera, sostiene que los niños no deben mostrar pasividad, silencio ni inmovilidad. $Y$ de acuerdo con su punto de vista, ese es el papel de los niños cuando se encuentran durante seis horas continuas frente a un televisor (Vallejo-Nagera,1988).

En relación con la apatía encontrada en los niños, Fernández-Collado y otros afirman que, cuando la ven diariamente durante 566 horas, "existe un efecto de letargo en el niño, quien mostrará gran pasividad y apatía hacia la realización de otras tareas" (Fernández-Collado, 1986:10).

La misma televisión que le impide al niño estudiar en sus tiempos libres actúa como una especie de droga a decir de algunos estudiosos del tema, incluso algunos de ellos emplean un término "teleadictos".

Winn (1981) describe de una manera interesante a los llamados teleadictos, afirma: "la experiencia de la televisión le permite al participante borrar el mundo real y penetrar en un estado mental pasivo y agradable. Las preocupaciones y 
ansiedades de la realidad quedan diferidas de una manera tan efectiva al concentrarse en un programa de televisión, como si emprendiera un viaje inducido por las drogas". Winn, además, afirma: "al igual que el alcohólico o el drogadicto, la televisión tiene el control sobre ellos, llegan a posponer actividades para disfrutar de los placeres de la televisión, las otras experiencias parecen menos atractivas y en cierta manera más difíciles" (Winn,1981:44).

\section{Posturas sobre el tema}

Otra de las posturas que existen en relación con el tiempo y el uso de la televisión por parte del niño sostiene que los niños cuando se encuentran por largos períodos de tiempo ante ese medio electrónico, tienden a desarrollar más un hemisferio cerebral que el otro. Esto es tienden a desarrollar más la habilidad para escuchar y ver que otro tipo de habilidades. En un congreso mundial relacionado con la educación preescolar celebrado en la ciudad de México, en 1988, se dieron cita destacados investigadores que han estudiado la relación de los medios de comunicación para con los niños. La Revista Mexicana de Comunicación invitó a varios de los participantes para que expusieran sus puntos de vista sobre la materia (Revista Mexicana,1989).

Beatriz Campos, subdirectora de Tiempo de niños dijo: "encuentro que uno de los efectos más grandes ha sido la uniformidad de los niños actuales. Sobretodo los que están expuestos a la televisión, no tienen la misma espontaneidad y frescura que aquéllos que no la ven con tanta frecuencia". Otra de las participantes, Patricia Navarrete hizo referencia a un estudio realizado, donde se midió el vocabulario de los adolescentes llegándose a la conclusión que éste ha disminuido.

"Se comprobó - dice la licenciada Navarrete - que ha disminuido la cantidad de palabras entre los adolescentes para expresar su cotidianeidad. Lo más grave es que todos utilizan frases ya hechas, y utilizan mensajes para la expresión de afectos, como si fueran parte de su identidad".

Navarrete expuso el descubrimiento de un científico soviético - no citó la fuente - el cual comprobó que la televisión afecta el hemisferio izquierdo del cerebro en los sujetos.

Al igual que la opinión anterior, Vallejo sostiene que los niños tienden a desarrollar más un hemisferio que otro, debido a la televisión, llegando a afirmar 
que una de las características presentadas por los malos estudiantes es su preferencia por ver la televisión durante largos períodos de tiempo.

Alejandra Vallejo encuentra entre los niños partidarios de ver mucha televisión: Malas notas escolares, inadaptación social, falta de esfuerzo, y una escasa participación con el profesorado (Vallejo-Nagera, 1988:88).

Al parecer los niños muestran una especie de apatía hacia las actividades ordinarias y exigen más dinamismo entre las acciones que desarrollan. Sauvage encontró que maestros de escuelas primarias de los Estados Unidos han modificado su forma de ofrecer clase. Cita - Sauvage - el caso de una maestra quien mencionó: "Ahora descubro que a cada rato tengo que ofrecer cosas que son de una gran actividad y que nunca antes tenía que ofrecer, porque los niños ya no persisten lo suficiente para descubrir si la cosa va a ser divertida en caso de que durante los primeros momentos no se diviertan" [...] "Hay niños que sencillamente se desconectan muy rápidamente" (Sauvage, 1972:118).

Otra maestra por su parte indicó, "he tenido que cambiar bastante mi estilo de enseñanza durante los últimos años, tengo la impresión de que ahora los niños quieren que yo tome la iniciativa", "simplemente prosiguen las actividades que yo inicio, y cuando no inicio nada, esperan con paciencia hasta que lo hago" (Sauvage,1972:120).

\section{Otras opiniones}

Entre las predicciones más alarmantes que existen sobre la televisión y los niños está seguramente la de Charles $\mathrm{A}$. Siepman. El realizó un estudio para la UNESCo titulado, $T$. V. y educación en los Estados Unidos.

Los comentarios emitidos por Siepman son los siguientes: "Veo acercarse la hora en que, como consecuencia de la televisión, el pueblo estadounidense no sabrá leer ni escribir y llevará una vida comparable con la de los vegetales" (citado en Cremoux, 1968). En 1962, Wilbur Schramm sostenía que los niños que no veían televisión al principiar a ir a la escuela, poseían un vocabulario más reducido y menos información en comparación con quienes estaban acostumbrados a verla.

Schramm, también encontró una reducción en las diferencias, y a la larga, los niños que iniciaron la escuela sin ver televisión, aventajaron a los niños que sí la veían en conocimientos sobre asuntos públicos, históricos y científicos, aprendiendo principalmente en la escuela (Schramm, 1961). 
A manera de resumen de este apartado se puede concluir que los niños dedican buena parte del día a ver la televisión y que de acuerdo con la opinión de varios especialistas en la materia, esta relación cuando se sostiene por grandes lapsos produce en los niños una especie de apatía hacia el mundo real, creando desinterés por cualquier otro tipo de actividades.

Finalmente, encontramos la opinión de otro grupo de investigadores quienes opinan que el frecuente uso del medio contribuye a desarrollar más un hemisferio que el otro.

\section{Método}

Para la realización del presente estudio, fueron considerados niños cuyas edades fluctuaron entre los 9 y los 12 años todos ellos residentes en los municipios de San Pedro Garza García, Guadalupe, San Nicolás de los Garza y Monterrey.

De acuerdo con el Ix Censo General de Población y Vivienda, la población total de la edad elegida es de 183,793 niños los cuales se encuentran distribuidos de la siguiente manera:

MUNICIPIO

San Pedro Garza García Guadalupe

San Nicolás de los Garza Monterrey

Total
POBLACIÓN

8,928

41,531

41,068

94,266

183,793

\section{Tamaño de la muestra}

Una vez identificado el número de niños que habitan el área metropolitana de Monterrey, Se procedió a calcular, el tamañode la muestra. Para lograrlo se recurrió a la siguiente fórmula. 


$$
n=\frac{2 p q}{\theta^{2}(N-1)+2 p q}=
$$

Previamente, fueron determinados los valores que se asignaron a cada uno de los elementos que conforman la fórmula:

$$
\begin{aligned}
& \theta^{2}=95 \% \\
& p=0.85 \\
& q=0.15 \\
& \theta^{2}=0.05 \% \\
& N=196 \text { niños }
\end{aligned}
$$

Tomando en cuenta los índices anteriores, el resultado sugiere un mínimo de 196 niños. Sin embargo el número de casos estudiado fue de 222 , es decir, 26 casos más de los exigidos.

Las características que se buscaron entre los encuestados fueron las siguientes:

1. Una edad de entre los 9 y 12 años.

2. Que fuesen estudiantes de nivel primaria.

3. Que estuvieran entre cuarto y sexto grado.

Sitio de la entrevista

En primer lugar se procedió a enumerar a cada una de las colonias del área metropolitana de Monterrey, tomando en cuenta que la mayor parte de éstas cuenta con al menos una escuela primaria dentro de su límite de territoriedad. Posteriormente por medio de las tablas con números aleatorios fueron seleccionados los sitios en donde habrían de ser aplicadas las 222 encuestas.

Cada uno de los encuestadores conocía el lugar exacto donde debería aplicar sus encuestas; el sexo y las características de sus entrevistados.

Los encuestadores en realidad aplicaron dos encuestas, la primera fue llenada con los datos proporcionados por los niños, mientras que la segunda correspondió a los datos aportados por el profesor del pequeño. 
El procedimiento fue el siguiente: una vez concluida la entrevista con los niños - los encuestadores - se dirigieron a la escuela donde estudiaba el pequeño. Ahí, contactaron al profesor, y le aplicaron la encuesta complementaria. ${ }^{2}$

\section{Resultados}

La muestra está constituida por un número mayor de niños que de niñas; mientras que el porcentaje de varones en el estudio es de 55\%, las niñas representan el $45 \%$.

La encuesta no contemplaba en especial un número determinado para niños que se encontraban estudiando en escuelas de tipo particular, sin embargo, la cifra de niños encuestados y que estudian en ese tipo de colegios corresponde al $14 \%$, contra un 86 de escuelas estatales y federales. Los resultados indican que el promedio de tiempo dedicado por los niños regiomontanos a ver la televisión es de 3 horas diarias. Esto significa que en promedio ven 566 programas diarios.

De los entrevistados, sólo 5\% dijo dedicar menos de una hora al día a ver televisión, contra más de una cuarta parte que mencionó verla más de tres horas y media, esto es, alrededor de 8 programas diarios.

En general los niños prefieren dedicar mayor cantidad de tiempo a la televisión durante la semana, en lugar de hacerlo durante el sábado o el domingo. Esto significa que ellos se sienten más atraídos por los programas que se presentan de lunes a viernes. Precisamente los días que tienen mayor obligación con sus actividades escolares. Sin embargo, pese a lo anterior, el 70\% de los niños negaron ver televisión al momento de realizar sus tareas. En este sentido sólo $12 \%$ de ellos reconocieron verla mientras elaboran sus tareas.

La realización del trabajo exigía de el cruce de variables para analizar los resultados. Por lo mismo se efectuó la operación cruzando las variables: "Clasificación del alumno", "promedio del alumno", " desempeño académico", "manejo del lenguaje”, promedios de Matemáticas, Ciencias Naturales, Ciencias Sociales, Español, contra la considerada por nosotros variable independiente "Tiempo de exposición a la televisión". 3 Para efectos del estudio esta última variable se dividió

2 En el caso de esta investigación, el desempeño escolar, el cumplimiento escolar, el promedio y las calificaciones de los niños fueron proporcionadas directamente por los profesores de los niños estudiados.

3 Estas fueron las materias que se manejaban en la currícula de primaria en la fecha del estudio. La modernización éducativa ha variado las materias recientemente. 
en cuatro categorías: en la primera se ubicó a quienes ven televisión menos de una hora al día; en la segunda a quienes la ven de 1 a 2:29; en la tercera están los niños que ven de 2:30 a 3:29 horas; finalmente en la cuarta categoría se incluyó a quienes dedican más de 3 horas y media diarias a ver televisión. Esta clasificación se utilizó en la mayoría de los análisis, excepto contra la variable "Manejo del Lenguaje". Una vez realizado el cruce de variables se utilizó en la totalidad de los casos la estadística Chi Cuadrada. En donde la significancia lo requirió, se procedió con la $\mathrm{C}$ de Contingencia para identificar el grado de relación entre las variables.

\section{Clasificación del estudiante}

Esta variable se clasificó de la siguiente manera: por una parte se encuentran los buenos y por la otra los malos estudiantes.

La primera categoría, se conformó por quienes habían sido clasificados como brillantes y buenos alumnos. La otra, la denominada malos, incluyó aquellos niños calificados como regulares, malos y pésimos estudiantes.

El cruce de tablas muestra que el promedio más bajo de niños clasificados como buenos, se encuentra precisamente entre quienes ven más de 3 horas y media la televisión (Cuadro 1). A la vez, el promedio más alto de estudiantes clasificados por sus maestros como malos, es el que se encuentra también entre quienes ven más de 3 horas y media la televisión.

Pese a los resultados, la Chi Cuadrada, no acepta la existencia de ningún vínculo entre las variables "Tiempo de Exposición" y "Clasificación del Estudiante".

\section{Cuadro 1.}

\begin{tabular}{|c|c|c|c|c|c|c|}
\hline \multirow{3}{*}{$\begin{array}{l}\text { HORAS DEDICADAS A VER } \\
\text { TELEVISIÓN, POR DIA }\end{array}$} & \multicolumn{4}{|c|}{ Clasificación } & \multirow{2}{*}{\multicolumn{2}{|c|}{ TOTAL }} \\
\hline & \multicolumn{2}{|c|}{ BUENO } & \multicolumn{2}{|c|}{ MALO } & & \\
\hline & No. & $\%$ & No. & $\%$ & No. & $\%$ \\
\hline $0: 00$ a $1: 00$ & 18 & 67 & 9 & 33 & 27 & 14 \\
\hline $1: 00$ a $2: 30$ & 31 & 67 & 15 & 33 & 46 & 24 \\
\hline $2: 30$ a $3: 30$ & 40 & 66 & 21 & 34 & 61 & 31 \\
\hline Más de 3:30 & 38 & 63 & 22 & 37 & 60 & 31 \\
\hline $\begin{array}{r}N=194 \\
X^{2}\end{array}$ & & & & 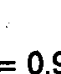 & 194 & 100 \\
\hline
\end{tabular}




\section{Desempeño del estudiante}

Esta segunda variable estudiada fue conceptualizada de la siguiente forma: primeramente se encuentran los niños muy cumplidos; después los niños considerados cumplidos. y finalmente están quienes cumplen regularmente.

En este caso los resultados muestran que los alumnos más incumplidos son los que ven menos de 1 hora al día la televisión. Se encuentra también, que los niños más cumplidos son quienes dedican de 1:00 a 2:30 horas diarias a verla (Cuadro 2).

Cuadro 2. Cumplimiento escolar

\begin{tabular}{|c|c|c|c|c|c|c|c|c|}
\hline \multirow[t]{2}{*}{ TIEMPO DE EXPOSICIÓN } & \multicolumn{2}{|c|}{ MUY CUMPLIDO } & & \multicolumn{2}{|c|}{ CUMPLIDO } & \multicolumn{3}{|c|}{ CUMPLE REGULAR } \\
\hline & No. & $\%$ & & No. & $\%$ & & No. & $\%$ \\
\hline $0: 00$ a $1: 00$ & 7 & 27 & & 7 & 27 & & 12 & 46 \\
\hline $1: 00$ a $2: 30$ & 17 & 37 & & 20 & 43 & & 9 & 20 \\
\hline $2: 30$ a $3: 30$ & 19 & 31 & & 22 & 36 & & 20 & 33 \\
\hline Más de 3:30 & 15 & 24 & & 29 & 47 & & 18 & 29 \\
\hline
\end{tabular}

$$
N=195 \text { X } X^{2}=7.82401 \quad .=6 \quad \propto=0.05 \quad P=7.733
$$

\section{Promedio en matemáticas}

En este caso fueron tomados en cuenta los promedios de los niños para ubicarlos dentro de cualquiera de las tres categorías previamente establecidas. Los alumnos cuyo promedio era superior a 9 fueron clasificados con la etiqueta bueno.

A quienes obtenían un promedio de 8 , se les denominó regular. Finalmente, el resto de pequeños fueron ubicados en una tercera categoría: malo, en ella están quienes tienen $7 \mathrm{o}$ menos de promedio. El resultado posterior al cruce de variables muestra que el promedio más alto de alumnos ubicado en la clasificación bueno está entre quienes dedican de 1:30 a 2:30 horas diarias a ver televisión (Cuadro 3).

El mismo cruce, pone de manifiesto que el promedio más alto de alumnos ubicados en la clasificación malo, es entre quienes dedican menos de una hora al día a ver televisión. En otras palabras, no son quienes dedican menor cantidad de 
tiempo a ver la televisión los mejores alumnos en Matemáticas, como originalmente se presuponía.

Para medir la relación que existe entre las variables se recurrió a la Chi Cuadrada, al igual que en los análisis anteriores. Y el resultado descarta toda posible relación entre las dos variables.

Cuadro 3. Promedio de calificaciones en matemáticas

\begin{tabular}{|c|c|c|c|c|c|}
\hline $\begin{array}{l}\text { TIEMPO DEDICADO A VER } \\
\text { TELEVISIÓN, POR DÍA }\end{array}$ & BUENO (\%) & REGULAR (\%) & MALO (\%) & $\begin{array}{l}\text { To } \\
\text { No. }\end{array}$ & AL \\
\hline $0: 00$ a $1: 00$ & 39 & 25 & 36 & 28 & 14 \\
\hline $1: 00$ a $2: 30$ & 52 & 22 & 26 & 46 & 23 \\
\hline $2: 30$ a $3: 30$ & 36 & 33 & 31 & 61 & 31 \\
\hline Más de 3:30 & 40 & 34 & 26 & 62 & 32 \\
\hline$N=197$ & 9103 & $\propto=0.05$ & $=0.6239$ & 197 & 100 \\
\hline
\end{tabular}

\section{Promedio en ciencias sociales}

Esta es una de las materias en donde los niños tienè promedios escolares más altos; en la materia sólo se presentan $3 \%$ de alumnos con calificaciones reprobatorias.

Para el análisis de esta variable se integraron tres categorías: Una integrada con niños cuyas calificaciones eran de 9 o 10, a ellos se les denominó bueno; la segunda conformada por quienes tenían de promedio 8 recibieron el nombre de regular; finalmente a los niños con calificaciones de 7 o inferiores, se les catalogó en la categoría denominada malo.

Los resultados de la operación indican que el mayor porcentaje de niños clasificados dentro de la categoría bueno, se encuentra entre quienes ven de una a 2:30 horas diarias la televisión.

La contraparte se da entre quienes dedican menor cantidad de tiempo diariamente a ver la televisión, pues ellos son quienes tienen ubicado un porcentaje mayor en la clasificación malo.

La aplicación de la Chi Cuadrada, sugiere la existencia de una relación entre las dos variables. Esto significa, que el tiempo que los niños ven la televisión, sí 
tiene que ver con el promedio que los niños obtienen en la materia de Ciencias Sociales.

Para conocer el grado de relación se procedió a utilizar la $\mathrm{C}$ de Contingencia. Dando por resultado que el grado de relación existente no sobrepasa el 30 por ciento (Cuadro 4).

Cuadro 4. Promedio de calificaciones en ciencias sociales

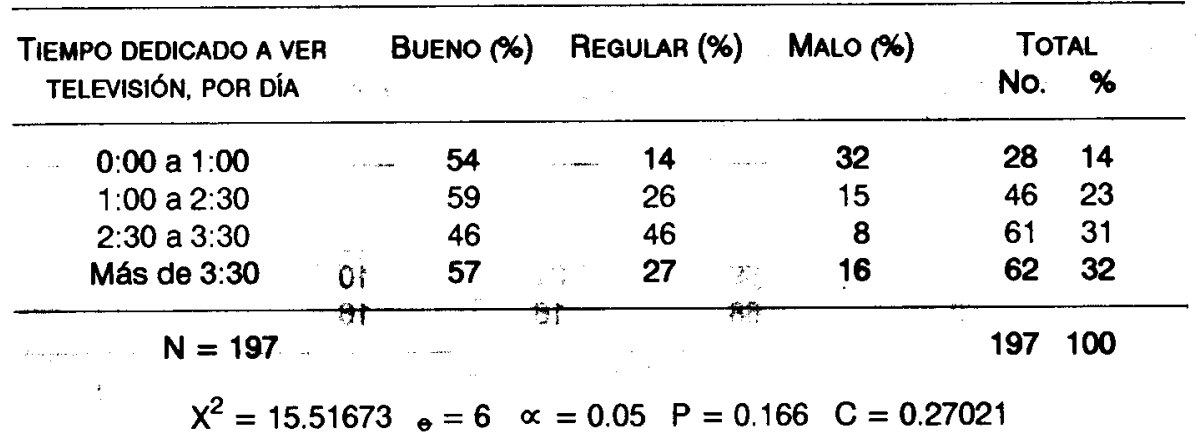

\section{Promedio en ciencias naturales}

En esta materia igual que en la anterior, son pocos los reprobados, sólo 4\% de los infantes se encuentran en esta situación. Y a decir de los maestros, dentro de esta materia no existen alumnos malos. Esta variable quedó conformada de la siguiente manera: la primera donde se incluyen a los alumnos cuyas calificaciones fueron superiores a 9, recibiendo el nombre de bueno; la segunda, se constituyó por alumnos con promedio de 8, denominándosele regular; finalmente, la llamada malo, se conformó con estudiantes cuyas calificaciones eran iguales o inferiores a 7 .

$\mathrm{Al}$ igual que en los análisis anteriores, se encuentra que no son mejores los alumnos que dedican menos tiempo diario a la televisión. La mayor cantidad o el mejor promedio que se encuentra en esta operación y ubicado dentro de la categoría bueno está conformado por niños que están frente al televisor de 1 a 2:30 horas diariamente (Cuadro 5). 
Nuevamente se encuentra lo que se manifestó anteriormente en el sentido de que aquellos estudiantes que ven menos de una hora diaria la televisión son los peores alumnos en la materia. El porcentaje mayor de alumnos ubicado dentro de la categoría malo corresponde a este tipo de personas. El resultado de la estadística Chi Cuadrada no confiere relación significativa entre las variables analizadas por lo tanto no puede considerarse el impacto de una variable sobre otra.

Cuadro 5. Promedio de calificaciones en ciencias naturales

\begin{tabular}{|c|c|c|c|c|c|}
\hline \multirow{2}{*}{$\begin{array}{l}\text { TIEMPO DEDICADO A VER } \\
\text { TELEVISIÓN, POR DIAA }\end{array}$} & \multirow[t]{2}{*}{ BUENO (\%) } & \multirow[t]{2}{*}{ REGULAR (\%) } & \multirow[t]{2}{*}{ MALO (\%) } & \multicolumn{2}{|c|}{ TOTAL } \\
\hline & & & & No. & $\%$ \\
\hline $0: 00$ a 1:00 & 39 & 39 & 22 & 28 & 14 \\
\hline $1: 00$ a $2: 30$ & 68 & 17 & 15 & 46 & 23 \\
\hline $2: 30$ a $3: 30$ & 59 & 31 & 10 & 61 & 31 \\
\hline Más de 3:30 & 68 & 16 & 16 & 62 & 32 \\
\hline$N=197$ & & & & 197 & 100 \\
\hline$\lambda$ & $\theta=6$ & $\propto=0.05$ & $=0.0804$ & & \\
\hline
\end{tabular}

\section{Promedio en la materia de español}

Para efectos del análisis de esta variable, se categorizó a la variable de tres distintas maneras: bueno, regular y malo, utilizándose para ello los mismos parámetros que las pruebas anteriores. De igual manera fue cruzada la variable mencionada contra el "tiempo de exposición a la televisión". Los resultados muestran nuevamente que no son los mejores alumnos los que dedican menos tiempo a ver televisión. El mayor porcentaje de alumnos ubicados dentro de la clasificación malo, se presenta entre quienes ven menos de una hora al día la televisión.

Esto significa en primera instancia, que los niños que ven la televisión "menos tiempo", tienen más problemas con esa materia. 
El empleo y aplicación de la Chi cuadrada, sin embargo, descarta que lo anterior sea aceptado, pues el resultado muestra una significancia muy por arriba de la aceptación establecida, descartándose de esa manera la posible relación (Cuadro 6).

Cuadro 6. Promedio de calificaciones en español

\begin{tabular}{|c|c|c|c|c|c|}
\hline $\begin{array}{l}\text { TIEMPO DEDICADO A VER } \\
\text { TELEVISIÓN, POR DÍA }\end{array}$ & BUENO (\%) & Regular (\%) & MALO (\%) & & TAL \\
\hline $0: 00$ a $1: 00$ & 61 & 14 & 25 & 28 & 14 \\
\hline $1: 00$ a $2: 30$ & 59 & 24 & 17 & 46 & 23 \\
\hline $2: 30$ a $3: 30$ & 62 & 25 & 13 & 61 & 31 \\
\hline Más de 3:30 & 48 & 32 & 20 & 62 & 32 \\
\hline$N=197$ & & & & 197 & 100 \\
\hline
\end{tabular}

\section{Manejo del lenguaje}

Los datos recabados indican que 63 por ciento de los niños tienen buen manejo del lenguaje; mientras que un 10 por ciento, lo maneja de manera brillante. Es decir tres cuartas partes de la muestra, no tienen ningún problema con el lenguaje.

A diferencia de los anteriores cruces de variables, en esta ocasión se determinó ubicar a los niños en dos categorías: En la primera se ubicaron a quienes tienen un brillante y buen manejo del lenguaje, ésta recibió el nombre de bueno. La segunda categoría, ubicó a quienes tienen un manejo del lenguaje regular y menos que regular, se le denominó malo.

A la vez el "Tiempo de Exposición" a la televisión, quedó finalmente conformado de la siguiente manera: El primer valor abarca de las 0:00 horas hasta 1:30 horas, el segundo abarca de 1:30 a 2:30 horas; la tercera categoría comprende de las 2:30 hasta las 3:30 horas la cuarta y última incluye a quienes ven más de 3:30 diariamente la televisión.

Los resultados indican que quienes ven televisión de una a 2:30 horas diariamente, son también quienes muestran mejor manejo del lenguaje. 
Mientras, que quienes tienen un peor manejo del lenguaje son aquellos que dedican mayor cantidad de tiempo - más de 3:30 horas - a ver televisión.

También el porcentaje de niños ubicado dentro de la clasificación denominada malo es menor entre quienes dedican de 1:30 a 2:30 horas diarias a la televisión. En este caso el resultado de la Chi cuadrada acepta que el "Tiempo de Exposición" a la televisión, sí influye en el manejo del lenguaje de la criatura.

El grado de relación existente entre las variables obtenido a través de $\mathrm{C}$ de Contingencia, sobrepasa el 20 por ciento (Cuadro 7).

Cuadro 7. Manejo del lenguaje

\begin{tabular}{|c|c|c|c|c|c|c|}
\hline \multirow{2}{*}{$\begin{array}{l}\text { TIEMPO DEDICADO A VER } \\
\text { TELEVISIÓN, POR DÍA }\end{array}$} & \multicolumn{2}{|c|}{ BUENO } & \multicolumn{2}{|c|}{ MALO } & \multicolumn{2}{|c|}{ TOTAL } \\
\hline & No. & $\%$ & No. & $\%$ & No. & $\%$ \\
\hline $0: 00$ a $1: 30$ & 18 & 67 & 9 & 33 & 27 & 14 \\
\hline $1: 30$ a $2: 30$ & 39 & 85 & 7 & 15 & 46 & 24 \\
\hline $2: 30$ a $3: 30$ & 50 & 82 & 11 & 18 & 61 & 31 \\
\hline Más de 3:30 & 37 & 61 & 24 & 39 & 61 & 31 \\
\hline$N=195$ & 144 & & 51 & & 195 & 100 \\
\hline
\end{tabular}

Promedio de los estudiantes

Esta variable es de mucha importancia para el estudio, pues el total de calificaciones de todas las materias están siendo estudiadas bajo este concepto.

La variable promedio se estructuró de la siguiente manera: primeramente a las calificaciones de 7 e inferiores se les catalogó en la categoría, malo. A quienes tenían promedio de 8 , se les ubicó en la casilla regular, por último, los promedios de 9 y 10 , integran la categoría bueno. El resultado del cruce de las variables "tiempo de exposición a la televisión" y "promedio de los estudiantes", indica que dentro de la categoría Bueno se encuentran más niños cuyo tiempo de exposición es inferior a la hora.

Los estudiantes que dicen ver televisión menos de una hora al día, son también quienes concentran menos alumnos ubicados en la categoría malo, esto tal vez 
podría responder la pregunta inicial de la investigación, sin embargo, el resultado de la estadística utilizada sobrepasaba el límite de significacia establecido, significando que la relación entre "tiempo de exposición a la televisión" y "promedio escolar", no se da (Cuadro 8).

Cuadro 8. Tiempo dedicado a ver televisión

\begin{tabular}{|c|c|c|c|c|c|c|c|}
\hline \multirow{2}{*}{ HORAS POR DíA } & \multirow[b]{2}{*}{ 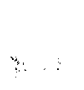 } & \multirow[t]{2}{*}{ BUENO (\%) } & \multirow{2}{*}{\multicolumn{2}{|c|}{ REgular (\%) }} & \multirow[t]{2}{*}{ MALO (\%) } & \multicolumn{2}{|c|}{ TOTAL } \\
\hline & & & & & & & $\%$ \\
\hline $0: 00$ a $1: 00$ & & 22 & & 39 & 39 & 28 & 14 \\
\hline $1: 00$ a $2: 30$ & & 20 & & 26 & 54 & 46 & 23 \\
\hline $2: 30$ a $3: 30$ & & 16 & & 38 & 46 & 61 & 31 \\
\hline Más de 3:30 & & 21 & 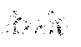 & 27 & 52 & 62 & 32 \\
\hline$N=197$ & & & & & & 197 & 100 \\
\hline & 3.435 & $3554 \Leftrightarrow=6$ & & $=0.05 \quad P=$ & 0.7525 & & \\
\hline
\end{tabular}

\section{Conclusiones}

Los resultados del trabajo muestran que, el ver mayor cantidad de tiempo la televisión no afecta el promedio de las calificaciones en las materias de Matemáticas, de Español, ni de Ciencias Naturales. La única materia donde sí se presenta relación entre las variables es la de Ciencias Sociales. Cabe destacar sin embargo, que el grado de relación existente es pequeño. Otra afectación se encuentra en el "manejo del lenguaje", donde ocurre, como en la anterior, una relación pequeña.

La pregunta con que se inició el presente estudio era: itienen los niños que dedican más tiempo a ver televisión, promedios de calificación más bajos que aquellos que le dedican menor cantidad de tiempo? Por lo tanto los resultados que ofrece la investigación están en contra de las predicciones y supuestos existentes sobre el tema. Al menos en este caso, bajo las condiciones metodológicas descritas en su momento, y con las técnicas estadísticas empleadas, se concluye que la televisión no interfiere de manera significativa en las calificaciones de los niños de escuelas primarias en Monterrey y su área metropolitana. 
Sobresale en los datos de la investigación el hecho de que los promedios más bajos corresponden a quienes ven televisión menor cantidad de tiempo. $Y$, quienes dedican un término medio de su tiempo a ver televisión son quienes tienen los promedios más altos.

\section{Referencias bibliográficas}

Acosta M. C. (1972): "Los niños mexicanos y la televisión". Revista Diálogos., Núm. 47. México, D. F., México.

AlMEIDA M., G. Villazón y M. GuTiérReZ (1977): "Funciones que desempeña la televisión para el niño mexicano". Tesis de licenciatura. Universidad Anáhuac, México, D. F., México.

Columna Editorial Fricase (1991): "Mal Educador". El Norte, Jueves 11 de Abril, p. 5A. Monterrey, Nuevo León, México.

CREMOuX Raúl (1968): La Televisión y el Niño de Secundaria del Distrito Federal. México, D. F.: Centro de Estudios Educativos, A.C.

“Culpa Bush a la televisión por baja educación" (1991): El Norte, Miércoles 4 de septiembre. p. 9A. Monterrey, Nuevo León, México.

Fernández-Collado Carlos, Pilar Lucio Baptista y Débora ElkEs (1986): La Televisión y el Niño. México: Editorial Oasis, Nueva Biblioteca Pedagógica 56.

FISCHER Laura y Alma NAVARRo (1984): Introducción a la Investigación de Mercados. México: Nueva Editorial Interamericana, S. A. de C. V.

"Indefinición de los Niños Frente a los medios de Comunicación Masiva" (1989): Revista Mexicana de Comunicación Año 2, Núm. 8. México.

INEGI (1991): XI Censo General de Población y Vivienda 1990. Aguascalientes, Ags.: Instituto Nacional de Geografía e Informática. 
Lozano José Carlos (1992): Media exposure and cultural identy in a Mexican border community, the case of "Secundaria" students. Disertación doctoral, Universidad de Texas en Austin.

MARTÍNEZ GARZA Francisco Javier (1992): Estudio de las audiencias infantiles de televisión. Monterrey, N.L.: Facultad de Ciencias de la Comunicación, Universidad Autónoma de Nuevo León.

MAS MARES Leticia Eugenia (1990): "T. V. Adicción". El Maestro, Año II, Núm.1. Monterrey, N. L., México.

MEZA Andrés (1991): "Estudio sobre la educación primaria en Monterrey y su área metropolitana".ElNorte, 7,8,9,10 de abril,Sección Cultural,p.1.Monterrey, N. L., México.

Orozco GómEz Guillermo (1991): "El Niño como televidente no nace se hace". Revista Andanzas Núm. 2. León, Gto.: Universidad Iberoamericana, plantel León.

SAUVAGE Nicole (1972): La televisión los Niños-la Familia. Bilbao, España: Ed. Mensajero, Colección Nuevo Horizonte.

SCHRAMM Wilbur (1961): Televisión in the lives of our childrens. Stanford,California: Stanford University Press.

VALlEJo-NAGERA Alejandra (1988): Mi Hijo ya no Juega Sólo ve la Televisión. ¿Qué puedo hacer?. México: Grupo Editorial Planeta, Ediciones Temas de Hoy.

WINN Marie (1981): La Droga que se enchufa. México: Editorial Diana. 